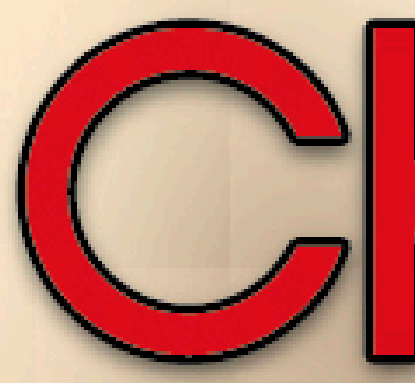

Revista Lattinoamericana de Comurnicación D
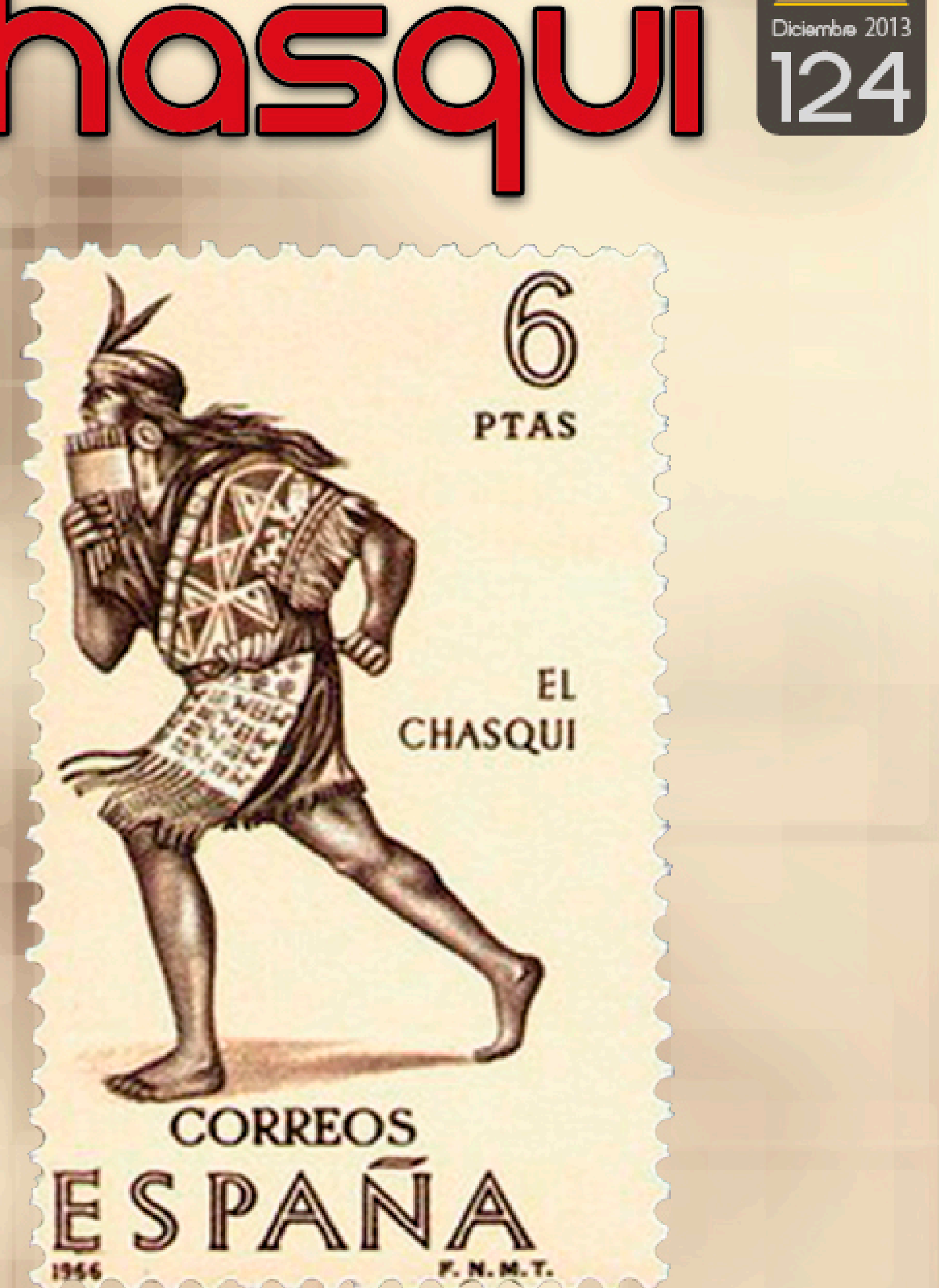

41 años $\cdot 2041$ artículos $\cdot 1332$ autores $\cdot 52$ países.

El rol social de la educación en medios 


\section{En esta edición}

\section{Tema central}

\section{El rol social de la educación en medios}

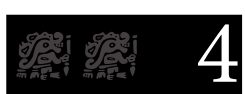

La urgencia de la competencia mediática en los programas escolares Alberto Parola Agueda Delgado Ponce

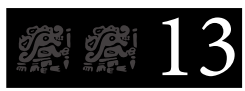

El desarrollo de la competencia mediática en personas mayores: una brecha pendiente Ángel Hernando Alejandra Phillippi

Presente y futuro de la alfabetización mediática en Europa:

el caso español y portugués Cristina Ponte

Paloma Contreras-Pulido

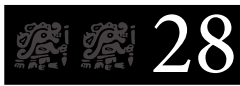

Avances para el desarrollo de la competencia mediática a partir del currículum de primaria en Colombia y España M. Amor Pérez-Rodríguez Yamile Sandoval

Las interacciones mediáticas de la juventud boliviana: la competencia mediática de jóvenes de La Paz

Vicent Gozálvez Pérez René Zeballos Clavijo

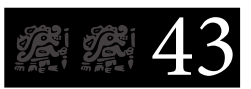

La competencia mediática en niños y jóvenes: La visión de España y Ecuador Isidro Marín-Gutiérrez Elena Díaz-Pareja Ignacio Aguaded

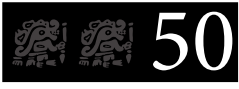

Competencia mediática ante la Agenda Setting: parámetros de actuación a nivel educativo Rosa García-Ruiz Diana Rivera-Rogel

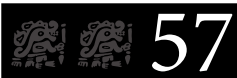

La alfabetización mediática en la televisión infantil online: programas del Canal Pakapaka Jacqueline Sánchez-Carrero Heleny Méndiz-Rojas

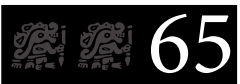

Educomunicación y radios universitarias: Panorama internacional y perspectivas futuras Ignacio Aguaded Daniel Martín-Pena

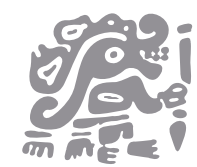




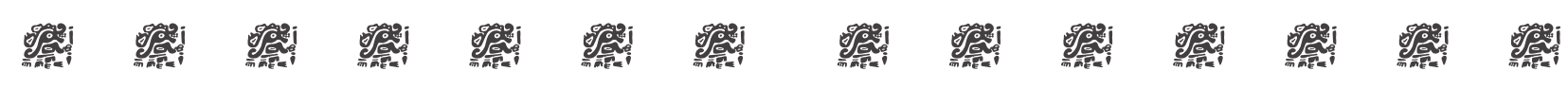

\section{Ensayo}

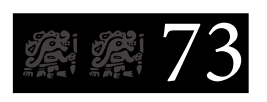

El sistema de comunicación en la Ley Orgánica de Comunicación del Ecuador: Un análisis desde el enfoque de las teorías de sociedad y masas de McQuail. Caroline Ávila

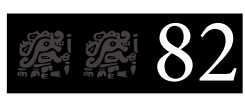

Periodismo y nuevas tecnologías: los ejes del pensamiento hegemónico y una posible contra agenda

Florencia Saintout

Redes de actores en medios de prensa.

Una metodología para abordar la hegemonía en los medios de comunicación: el ejemplo de las elecciones de 2013 en Paraguay. Rubén Juste de Ancos

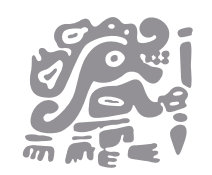

\section{Informe}

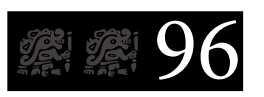

La colonización de la mirada. Un análisis de la información internacional en la prensa puertorriqueña Israel Rodríguez Sánchez

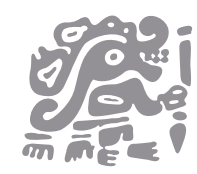

\section{Visual}

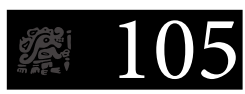

Miceláneos al fin del 2013

Diego S Acevedo

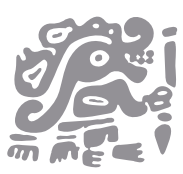

\section{Reseñas}

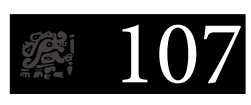

Anuario Obitel 2013

Memoria social y ficción televisiva en Iberoamérica

Alexandra Ayala Marín

Chasqui es un espacio para el desarrollo y difusión del pensamiento crítico en torno a la relación comunicación, cultura y política en el ámbito latinoamericano. Participan académicos, escritores, periodistas, artistas y otros pensadores unidos por la necesidad común de proponer, desde la comunicación, ideas impugnadoras al pensamiento dominante y al poder. 


\section{La educación en medios}

La Declaración de Grünwald, supuso el punto de partida para promover entre las autoridades competentes «programas integrados de educación relativa a los medios de comunicación desde el nivel preescolar hasta el universitario y la educación de adultos» (UNESCO, 1982). Objetivo que aparece y se mantiene constante desde muchas iniciativas gubernamentales ${ }^{1}$ a lo largo de las dos últimas décadas. Desde entonces, se han consolidado una serie de propuestas para contribuir a la educación en medios, tales como promover la formación de docentes, formadores y profesionales de los medios de comunicación, integrar la alfabetización mediática en los programas escolares, invertir en la producción de recursos para apoyar la alfabetización mediática, entre otros.

La alfabetización y educación mediática se convierten en una prioridad para la UNESCO, (2007, 2011), que marca entre sus objetivos preferentes el desarrollo de una educación crítica, activa y plural ante los medios de comunicación.

\section{Colombia}

El Plan Decenal de Educación (PDNE) 2006-2015 reconoce oficialmente la influencia de los medios en la educación de los niños, especialmente la televisión enfatizando en la necesidad de asumir compromiso desde los diferentes sectores involucrados en la educación y programar responsablemente los contenidos. Los medios de comunicación posibilitan procesos de formación efectivos y desde el PNDE se aboga por mayores espacios que favorezcan el aprendizaje para el fomento de la cultura de paz y los valores. De

\footnotetext{
1 El coloquio de Toulouse «Nuevas direcciones en educación en medios» de 1990; la Conferencia de Viena «Educando para la era digital» en 1999; la iniciativa «eEurope» de 2000; el seminario «Educación en Medios para Jóvenes» celebrado en Sevilla en 2002; la Cumbre Mundial sobre la Sociedad de la Información de 2003, en Ginebra; la Proclamación de Alejandría acerca de «la Alfabetización Informacional y el Aprendizaje de por Vida» de 2005; la Carta Europea para la Alfabetización en Medios publicada en 2006; el Digital Literacy High-Level Experts Group desarrollado en 2006; la Directiva de Servicios de Medios Audiovisuales de 2007; las Doce Recomendaciones para la Educación Mediática de la Agenda de París en 2007; la Recomendación de 2009 de la Comisión Europea sobre la alfabetización mediática en el entorno digital para una industria audiovisual y de contenidos más competitiva y una sociedad del conocimiento incluyente; la Declaración de Bruselas por una «Éducation aux Médias tout au long de la vie» en 2011; y la Declaración de Braga de 2011
}

igual forma, la ley 1098 de noviembre del 2006, presenta el Código de la Infancia y la Adolescencia, que delega responsabilidades especiales de los medios de comunicación.

En Colombia la educación en medios la dinamizan maestros apasionados, quienes maestros apasionados, quienes especialmente desde las áreas de Lenguaje y comunicación, han liderado proyectos que involucran productos de los medios de comunicación en su contexto de enseñanza y aprendizaje, y aunque esta relación es una forma inicial de integración entre el currículo y la educación en medios (Gutiérrez y Tyner, 2012) no avanza significativamente.

Por otra parte, se han llevado a cabo programas independientes como «Prensa Escuela», diseñado desde los productores de prensa escrita para acercar al estudiante con la lógica del medio, o de la academia con proyectos de formación de públicos como "Mirando cómo Miramos», desarrollado a lo largo de seis años con el objetivo de formar televidentes críticos, activos y socialmente responsables.

\section{España}

Uno de los primeros pasos para la educación en medios se produce al plantearse la Ley 7/2010 General de la Comunicación Audiovisual ${ }^{2}$ que regula los contenidos de los medios de comunicación para «proteger» a niños y jóvenes, además de velar por los derechos de los telespectadores.

El Plan Estratégico Nacional de Infancia y Adolescencia (2006-2009)3, aboga por el conocimiento y utilización de los medios de comunicación y las tecnologías como instrumentos de aprendizaje y desarrollo entre niños y jóvenes, además de incluir medidas para la protección, fomentar una visión crítica y participativa de la televisión y facilitar el acceso responsable y generalizado a Internet.

Programas como «Prensa-Escuela» del Ministerio de Educación, dirigido a los Centros Educativos, para la introducción de los periódicos en el aula ${ }^{4}$,

\footnotetext{
2 www.boe.es/boe/dias/2010/04/01/pdfs/BOE-A-2010-5292. pdf

$3 \mathrm{http}: / / \mathrm{tv} \_$mav.cnice.mec.es/pdf/Plan_Estrat_Inf_Ad.pdf

4 www.educacion.gob.es/documentos/mediascopio/archivos_ secciones/156/Carpetas1.Parte1[1]. Pdf.
} 
Atenea y Alhambra para el uso de las nuevas tecnologías y la informática, o Mercurio para la utilización didáctica de los medios audiovisuales, junto a la producción audiovisual «Televisión Educativa: la Aventura del Saber $»^{5}$, constituyen importantes iniciativas en este campo. Mientras que a nivel educativo se imparten asignaturas en Secundaria y Bachillerato como «Imagen y expresión», «Procesos de comunicación» y «Comunicación audiovisual».

Sin embargo, las experiencias más

La alfabetización necesaria hoy hay que considerarla como el desarrollo de una competencia digital $y / o$ audiovisual que convergen en lo que podría considerarse la competencia mediática destacadas en este campo se realizan mediante proyectos, muchas veces no amparados por las instituciones, para la educación en medios ${ }^{6}$.

En la actualidad,
la inclusión entre
las competencias
básicas del sistema
educativo vigente,
de «el Tratamiento de la información y competencia digital», incorpora la alfabetización mediática al contemplarse para el desarrollo de ésta el dominio de los diferentes lenguajes y soportes y su aplicación a la comprensión, la interpretación crítica, la comunicación y la expresión, recogiendo conceptos como el de educación en materia de comunicación o educación para la comunicación crítica (Aparici, Campuzano, Ferrés y GarcíaMatilla, 2010).

Finalmente en el terreno de la investigación el Proyecto de I+D «La competencia en

5 Programa diario matinal de 1 hora de duración destinado a la formación y la divulgación mediante entrevistas y series documentales. www.rtve.es/television/la-aventura-del-saber/

6 Drac Magic, de Barcelona; Aire, de Madrid; Spectus, de Aragón; Mitjans y Análisis de la Recepción de las Pantallas Audiovisuales (ARPA), de Cataluña; Apuma, de carácter estatal; y Eko Kolektiboa, del País Vasco. También sobresalen iniciativas como Pé de imaxe, en Galicia, o Cinema Jove, en Valencia, así como la labor desarrollada por el Instituto de Tecnologías Edu-cativas (ITE) del Ministerio de Educación y el Instituto Radiotelevisión (IORTV-RTVE), de la televisión pública estatal, Gabinete de Comunicación y Educación de la Autónoma de Barcelona y el Grupo Comunicar y la Universidad de Huelva. comunicación audiovisual en un entorno digital. Diagnóstico de necesidades en tres ámbitos sociales», abandera la detección de necesidades y carencias en torno a la competencia en comunicación audiovisual de la ciudadanía, así como la proyección de propuestas de alfabetización mediática ${ }^{7}$.

\section{La competencia mediática}

Dada la profusión de medios, el avance y progreso tecnológico, las brechas que se originan intergeneracionales, la tan traída y llevada metáfora de los nativos digitales (Prensky, 2000), la educación en medios se dibuja como un objetivo obligado. Aguaded y Sandoval (2011), a partir de «la convergencia de múltiples pantallas con diversos, simultáneos e instantáneos contenidos de todo orden, disponibles a través de la red», plantean una educación en medios, que abandone la visión instrumental del medio de comunicación como aparato reproductor y comprenda las dinámicas de un nuevo «prosumidor».

Por tanto, y ante la tendencia de los actuales medios hacia la digitalización, la alfabetización necesaria hoy hay que considerarla como el desarrollo de una competencia digital y/o audiovisual que convergen en lo que podría considerarse la competencia mediática (Comisión Europea, 2011, p. 14; Pérez-Rodríguez \& Delgado, 2012). En este sentido, ésta se entiende como la capacidad de percibir, analizar y disfrutar del poder de los mensajes, imágenes, estímulos y sonidos que percibimos de los medios de comunicación, usándolos para satisfacer nuestras necesidades de comunicación, expresión, formación o información que puedan generarse en diversas situaciones cotidianas (Comisión Europea, 2011).

Para su desarrollo contemplamos diez dimensiones siguiendo a Pérez-Rodríguez y Delgado (2012, p. 32). El ámbito del conocimiento que comprendería: Política e industria mediática, Procesos de producción, Tecnología, Lenguaje, Acceso y obtención de información. El ámbito de la comprensión que reuniría las dimensiones de: Recepción y comprensión e Ideología y

\footnotetext{
7 En él participan la Universidad Pompeu Fabra, la Universidad de Huelva y la Universidad de Valladolid, en una labor conjunta para contribuir al desarrollo de la competencia mediática. Más información en: http://www.competenciamediatica.es/
} 
valores. Finalmente, en la cúspide, la expresión, se situaría: Comunicación, Creación y Participación ciudadana.

\section{Análisis de la competencia mediática en el currículum de Primaria}

Para llevar a cabo este análisis, se han revisado los documentos curriculares de ambos países en relación con la Etapa de Primaria y el área de Lengua, por ser ésta una de las más vinculadas al desarrollo de la competencia mediática, considerando las dimensiones para el tratamiento de la competencia mediática expuestas por PérezRodríguez y Delgado (2012).

Se pretende la revisión de lo prescrito en el currículum al objeto de determinar si la propuesta curricular de cada país garantiza o potencia el desarrollo de la competencia mediática, especialmente en el Área de Lengua/Lenguaje, así como determinar qué dimensiones se abordan.

\section{Colombia}

Conscientes de la importancia de formar frente a los medios de comunicación y los mensajes que estos ponen en circulación, los indicadores de la competencia mediática han sido incluidos con especial énfasis en los estándares básicos del Lenguaje y de manera posterior en el 2008, en lo que denominaron desde el Ministerio de Educación «Orientaciones generales para la educación en tecnología».

La Educación Básica se divide en dos grupos de grados, de acuerdo a la estructura del sistema educativo colombiano: primero a tercer grado, y cuarto a quinto grado. Las competencias se enuncian como transversales a las áreas del currículo y del conocimiento y son desarrolladas en trabajos concretos. Se concibe su desarrollo en "afinidad con los procesos de desarrollo biológico y psicológico del estudiante. Es en este sentido que se habla de una coherencia vertical» (MEN, 2008, p.15).

Los medios de comunicación, se incluyen dentro del lenguaje no verbal, en uno de los tres campos de la formación en Lenguaje, denominado «Pedagogía de otros sistemas simbólicos» ${ }^{8}$. Para el

8 En el documento, se proponen tres campos de la formación en lenguaje: La pedagogía de la lengua castellana; la pedagogía estándar, se propone una estructura conformada por: Factores. En Lenguaje: Producción textual; comprensión e interpretación de textos; Literatura; Medios de comunicación y otros sistemas simbólicos, y Ética de la comunicación. Un enunciado identificador, que concreta la competencia específica y, Los subprocesos, que se refieren a indicadores de logro concretos.

En relación a la primera dimensión Acceso y búsqueda de información, en los enunciados de Producción textual y comprensión e interpretación textual, y en el de Medios de Comunicación y otros sistemas simbólicos -en todos los grados- se encuentran referencias en línea con el conocimiento de los procesos de búsqueda, las normas de acceso y la capacidad para extraer información de diversas fuentes. Asíse expresan como subprocesos: «Busco información en distintas fuentes: personas, medios de comunicación y libros, entre otras», "Determino algunas estrategias para buscar, seleccionar y almacenar información...», "Utilizo estrategias de búsqueda, selección y almacenamiento de información para mis procesos de producción y comprensión textual», "Utilizo los medios de comunicación masiva para adquirir información e incorporarla de manera significativa a mis esquemas de conocimiento» o "Selecciono y clasifico la información emitida por los diferentes medios de comunicación»

La dimensión Lenguaje, está asociada a los factores: Medios de comunicación y otros sistemas simbólicos, y Ética de la comunicación. Se centra en el reconocimiento de los códigos utilizados; las estructuras narrativas; los actores dentro del proceso; la comparación entre los textos y su complementariedad, para de manera integral darle un sentido al texto en el contexto en que éste se utiliza. Se muestran, entre otros, los subprocesos de: «Caracterizo algunos medios de comunicación: radio, televisión, prensa...», «Identifico la información que emiten los medios de comunicación masiva y la forma de presentarla», «Establezco diferencias y semejanzas entre noticieros, telenovelas, anuncios comerciales, caricaturas...», «Entiendo el lenguaje empleado en historietas y otros tipos de textos con imágenes fijas», "Ordeno y completo la secuencia de viñetas que conforman una historieta» y «Reconozco

de la literatura y la pedagogía de otros sistemas simbólicos. Estándares Básicos de Competencias del Lenguaje. Ministerio de Educación, 2006, Colombia. 
las características de los diferentes medios de comunicación masiva».

Ideología y valores se visibiliza en: «Identifico en situaciones comunicativas reales los roles, las intenciones de los interlocutores y el respeto por los principios básicos de la comunicación», «Identifico la intención de quien produce un texto» $\mathrm{o}$ «ldentifico en situaciones comunicativas reales los roles de quien produce $y$ de quien interpreta un texto», en los que se muestra la capacidad de estimar la objetividad y fiabilidad de las informaciones procedentes de los distintos medios, los intereses y valores que subyacen en determinados mensajes o la realidad y la representación que los medios ofrecen de ella.

La dimensión Recepción y comprensión, se desarrolla de manera transversal, asociada a los factores de Medios de comunicación y otros sistemas simbólicos y Ética de la comunicación. Los subprocesos se identifican con la comprensión de la información procedente de diferentes medios de comunicación y tecnología, la capacidad de reconocer por qué gustan o atraen determinados mensajes o la capacidad para diferenciar entre la emoción que provocan algunos mensajes audiovisuales y las valoraciones racionales que se hacen de ellos: «Identifico los diversos medios de comunicación masiva con los que interactúo», «Comento mis programas favoritos de televisión o radio», "Reconozco la temática de caricaturas, tiras cómicas, historietas, anuncios publicitarios y otros medios de expresión gráfica», «Identifico en situaciones comunicativas reales los roles de quien produce y de quien interpreta un texto».

El resto de las dimensiones consideradas en nuestro análisis no están presentes en los estándares básicos del Lenguaje. Sin embargo sí se encuentran en el desarrollo de orientaciones para ser competentes en Tecnología. Este componente adicionando a los estándares existentes (MEN, 2008, p. 5), se estructura como: 1) Componentes: naturaleza y evolución de la tecnología; apropiación y uso; solución de problemas con tecnología, y, tecnología y sociedad. 2) Competencias, y 3) Desempeños, que dan cuenta de la evolución para alcanzar la competencia a lo largo del recorrido escolar. (MEN, 2008, p.15).

Demanera transversal se desarrolla la dimensión de Recepción y comprensión, cuando el sentido de esta orientación es hacer conscientes a los estudiantes del artefacto y analizar crítica y reflexivamente su articulación con la sociedad. Dentro del mismo estándar se despliegan indicadores de las dimensiones de tecnología; comunicación; participación ciudadana, y política e industria mediática. Sirvan como ejemplo: «ldentifico la computadora como artefacto tecnológico para la información y la comunicación, y la utilizo en diferentes actividades», «Manifiesto interés por temas relacionados con la tecnología a través de preguntas e intercambio de ideas», «Participo en equipos de trabajo para desarrollar y probar proyectos que involucran algunos componentes tecnológicos», «Participo con mis compañeros en la definición de roles y responsabilidades en el desarrollo de proyectos en tecnología», e «Identifico instituciones y autoridades a las que puedo acudir para solicitar la protección de los bienes y servicios de mi comunidad».

\section{España}

La competencia mediática, está presente en la educación obligatoria española a partir de la Ley Orgánica de Educación (LOE) ${ }^{9}$, recomendándose en la Etapa de Educación Primaria, «sin perjuicio de su tratamiento específico en algunas de las áreas de la etapa, la comprensión lectora, la expresión oral y escrita, la comunicación audiovisual, las tecnologías de la información y la comunicación y la educación en valores se trabajarán en todas las áreas» (art. 19.2). Además, entre sus objetivos generales se incluye «Iniciarse en la utilización, para el aprendizaje, de las tecnologías de la información y la comunicación desarrollando un espíritu crítico ante los mensajes que reciben y elaboran» (MEC, 2006).

En el caso del Área de Lengua y Literatura se encuentran referencias directas a la competencia mediática entre sus objetivos: «Usar los medios de comunicación social y las tecnologías de la información y la comunicación, para obtener, interpretar y valorar informaciones y opiniones diferentes» (MEC, 2006), y en los contenidos de los bloques referidos a: Escuchar, hablar y conversar, leer y escribir, de cada uno de los ciclos.

La dimensión, Acceso y búsqueda de información, entendida como conocimiento de los procesos de búsqueda, las normas de acceso y la capacidad para extraer información de diversas fuentes,

9 www.boe.es/boe/dias/2006/05/04/pdfs/A17158-17207.pdf. 
es la más referenciada. Así, se ve en contenidos en los que se ha de abordar la «comprensión y valoración de textos orales procedentes de la radio y la televisión para obtener información general sobre hechos y acontecimientos próximos a la experiencia infantil», en el primer ciclo; o de «textos orales procedentes de la radio, la televisión o Internet con especial incidencia en la noticia, para obtener información general sobre hechos y acontecimientos que resulten significativos», en el segundo y tercero. Es importante destacar cómo se plantea, tanto en el segundo como en el tercer ciclo el «Uso de documentos audiovisuales para obtener, seleccionar y relacionar informaciones relevantes (identificación, clasificación, comparación)», algo que lleva implícito además del conocimiento de los procesos de búsqueda (definición de tópicos, conectores), la capacidad para extraer información de diversas fuentes, adecuada a las distintas situaciones comunicativas. También se especifican contenidos vinculados con destrezas de búsqueda y procesamiento de la información en relación con la lectura y escritura: «Utilización dirigida de las tecnologías de la información y la comunicación y de las bibliotecas para obtener información y modelos para la composición escrita», «utilización dirigida de las tecnologías de la información y la comunicación para la localización, selección y organización de información» $y$ «uso de las bibliotecas, incluyendo las virtuales, de forma cada vez más autónoma, para obtener información y modelos para la producción escrita».

En relación con la dimensión del Lenguaje, tanto a nivel de expresión como de comprensión oral y escrita, se exponen en el primer y segundo ciclo contenidos como: "comprensión y valoración de textos orales procedentes de la radio y la televisión», «comprensión de informaciones audiovisuales procedentes de diferentes soportes», «composición de textos de información y opinión propios de los medios de comunicación social sobre acontecimientos significativos, con especial incidencia en la noticia y en las cartas al director, en situaciones simuladas o reales». Se atiende así al conocimiento de los códigos del lenguaje mediático y a la capacidad de combinary establecer relaciones de complementariedad entre ellos, así como a los tipos o géneros de los mensajes: «comprensión de textos orales procedentes de la radio, de la televisión o de Internet con especial incidencia en la noticia, la entrevista, el reportaje infantil y los debates y comentarios de actualidad, para obtener información general sobre hechos y acontecimientos que resulten significativos y distinguiendo información de opinión», «Utilización dirigida de las tecnologías de la información y la comunicación» y «composición de textos de información y opinión».

Tecnología aparece como conocimiento de los sistemas informáticos y del sistema operativo y manejo de las herramientas necesarias para llevara cabo la comunicación. Puede verse en contenidos como: «utilización guiada de programas informáticos de procesamiento de texto», o el ya mencionado de «Utilización dirigida de las tecnologías de la información y la comunicación», «uso de las bibliotecas, incluyendo las virtuales», y «Utilización progresivamente autónoma de programas informáticos de procesamiento de texto». No se contemplan capacidades relacionadas con manejar las tecnologías para la creación de documentos multimedia.

En cuanto a la dimensión referida a los procesos de producción, se presenta en contenidos tales como: «Producción de textos orales propios de los medios de comunicación social mediante simulación o participación para ofrecer y compartir información y opinión», en el tercer ciclo, y aquellos en los que se alude a la composición, por lo que implican de conocimiento de las fases en las que se descomponen los procesos de producción y programación, o a las diferencias fundamentales entre las emisiones en directo y en diferido en los distintos medios.

Ideología y valores se muestra en relación con la capacidad de estimar la objetividad y fiabilidad de las informaciones procedentes de los distintos medios, detectar los intereses y valores que subyacen en determinados mensajes, distinguir entre la realidad y la representación que los medios ofrecen de ella, en contenidos como: "comprensión de textos orales procedentes de la radio, de la televisión o de Internet con especial incidencia en la noticia, la entrevista, el reportaje infantil y los debates y comentarios de actualidad, para obtener información general sobre hechos y acontecimientos que resulten significativos y distinguiendo información de opinión», «valoración de los medios de comunicación social como instrumento de aprendizaje y de acceso a informaciones y experiencias de otras personas» $y$ «composición de textos de información y opinión propios de los medios de comunicación social 
sobre acontecimientos significativos, con especial incidencia en la noticia y en las cartas al director, en situaciones simuladas o reales».

La dimensión Recepción y comprensión, aparece en muchos de los contenidos ya indicados, en relación con la comprensión de la información procedente de diferentes medios de comunicación y TIC, como en «comprensión y valoración de textos orales procedentes de la radio y la televisión...» o «comprensión de información general en textos procedentes de medios de comunicación social (incluidas webs infantiles) con especial incidencia en la noticia y en las cartas al director, localizando informaciones destacadas en titulares, entradillas, portadas...». Sin embargo no se atiende a la capacidad de reconocerse como audiencia activa, especialmente a partir del uso de las tecnologías digitales que permiten la participación y la interactividad, o al reconocimiento de por qué gustan o atraen determinados mensajes o a la diferencia entre la emoción que provocan algunos mensajes audiovisuales y las valoraciones racionales que se hacen de ellos.

Finalmente, la dimensión Comunicación, vinculada a la capacidad para comunicarse eficazmente a través de las distintas herramientas y soportes, y a la colaboración en la realización de proyectos a distancia utilizando los entornos digitales, se muestra en «valoración de los medios de comunicación social como instrumento de aprendizaje y de acceso a informaciones y experiencias de otras personas» y «producción de textos orales propios de los medios de comunicación social mediante simulación o participación para ofrecer y compartir información y opinión».

No se contemplan las dimensiones Participación ciudadana, es decir, capacidad para el ejercicio responsable de la ciudadanía digital, capacidad de interaccionar con las pantallas o responsabilidad cívico-política para llevar a cabo las acciones correspondientes ante el incumplimiento de las normas vigentes en materia audiovisual; y Creación, o habilidades para crear documentos multimedia, elaborar vídeos, montajes de sonidos e imágenes, utilizando adecuadamente los distintos códigos, creación de páginas web, blog, wikis usando las herramientas tecnológicas disponibles en Internet o trabajos originales utilizando los medios de comunicación y las TIC. Se alude a composición de textos de índole lingüística y en formato escrito con el uso de procesadores.

\section{Conclusiones}

Una vez revisado lo prescrito en el currículum/ estándares de cada país, queda patente la importancia de la competencia mediática, al comprobarse el tratamiento en ambas propuestas curriculares. Si bien son diferentes planteamientos, hay rasgos comunes como la consideración sobre todo de los medios y tecnologías como recursos para la búsqueda de información, la atención a la comprensión de los mensajes y usos de los medios, el conocimiento de los códigos y géneros, la consideración de las intenciones, o la ideología y valores. De alguna manera es un planteamiento lógico cuando la materia tiene que ver con la comunicación lingüística. Resulta interesante la propuesta colombiana que define un factor específico en el área de lenguaje para los medios de comunicación, sin embargo, se incluye como un instrumento dentro del proceso y no como un elemento con lenguaje propio y posibilidades para crear nuevo conocimiento.

El currículum colombiano al plantear transversalmente orientaciones para ser competentes en Tecnología permite abordar las otras dimensiones más específicas y necesarias para una verdadera alfabetización mediática, Comunicación, Participación ciudadana y Creación.

Es claro que en los dos países, los mayores aportes se hacen desde lugares distintos al currículum, lo que nos lleva a ahondar más adelante en proyectos para desarrollar y evaluar las competencias propuestas en la práctica.

\section{Bibliografía}

Aparici, Roberto, Campuzano, Antonio, Ferrés, Joan y García-Matilla, Agustín (2010). La educación mediática en la escuela 2.0. (www.airecomun.com/ sites/all/files/materiales/educacion_mediatica_ e20_julio20010.pdf). 
Comisión Europea (2006). Report on the results of the public consultation on Media Literacy. (http:// ec.europa.eu/culture/media/media-literacy/ public-consultation-on-media-literacy_en.htm).

Comisión Europea (2007). Comunicación de la Comisión al Parlamento Europeo, al Consejo, al Comité económico y social europeo y al Comité de las regiones «Un planteamiento europeo de la alfabetización mediática en el entorno digital». (http://ec.europa.eu/culture/media/literacy/docs/ com/es.pdf).

Comisión Europea (2009). Commission Recommendation, 20.8.2009, on media literacy in the digital environment for a more competitive audiovisual and content industry and an inclusive knowledge society. Recuperado de (http://ec.europa. eu/culture/media/media-content/medialiteracy/c_2009_6464_en.pdf).

Gutiérrez, Alfonso y Tyner, Kathleen (2012). Alfabetización mediática en contextos múltiples. Comunicar, 38, 10-12. (DOI: 10.3916/C38-2012-02-00).

MEC (2006). Real Decreto 1513/2006, de 7 de diciembre, por el que se establecen las enseñanzas mínimas de la Educación Primaria. BOE, 293 (08/12/2006). Madrid: Ministerio de Educación.

MEN (2008). Ser competente en tecnología: una necesidad para el desarrollo. Bogotá: Ministerio de Educación Nacional. (www.mineducacion.gov.co/1621/ articles-160915_archivo_pdf.pdf).

MEN (2006). Estándares básicos de competencias en Lenguaje, Matemáticas, Ciencias y Ciudadanía. Guía sobre lo que los estudiantes deben saber y saber hacer con lo que aprenden. Bogotá: Ministerio de Educación Nacional. (www.mineducacion.gov. co/1621/articles-116042_archivo_pdf.pdf).

Pérez-Rodríguez, M. Amor \& Delgado, Águeda (2012). De la competencia digital y audiovisual a la competencia mediática: dimensiones e indicadores. Comunicar, 39, 25-34. (DOI: 10.3916/C39-2012-02-02).

Prensky, Marc (2001). Digital Natives, Digital Immigrants, Part II: Do They Really Think Differently? Published in On the Horizon (NCB University Press, 9, 6, December 2001) (www.marcprensky.com/ writing/prensky\%20-\%20digital\%20natives, $\% 20$ digital\%20immigrants\%20-\%20part2.pdf).

Sandoval, Yamile \& Aguaded, José Ignacio (2012). Nuevas audiencias, nuevas responsabilidades. Icono14. ISSN 1697-8293. Volumen 10 No.3. Pp.08-22. (DOI: 10.7195/ri14.v10i3.197). (www.icono14.net/ Numero-actual).

UNESCO (1982). Declaración de Grünwald sobre la educación relativa a los medios de comunicación. (www.unesco.org/education/pdf/ media_s.pdf).

UNESCO (1999). Conferencia «Educating for the Media and the Digital Age». (http://edu.of.ru/attach/17/3485. PDF).

UNESCO (2007). Agenda de París o 12 Recomendaciones para la Educación en Medios. (www.ifap.ru/ pr/2007/070625ba.pdf).

UNESCO (2011). Declaración de Braga. (www.cca.eca.usp. $\mathrm{br} /$ noticia/756). 